\title{
Interpersonal Aspects of Responsibility and Obsessive Compulsive Symptoms
}

\author{
Andrea R. Ashbaugh, Laurie A. Gelfand and Adam S. Radomsky \\ Concordia University, Montreal, Canada
}

\begin{abstract}
Obsessive-compulsive disorder (OCD) is associated with an inflated sense of responsibility to prevent harm. Increasingly, it has been recognized that inflated responsibility is a complex phenomenon. The purpose of this study was to examine how interpersonal aspects of responsibility are related to symptoms of OCD. Three new valid and reliable scales assessing beliefs about other people's responsibility, comparative beliefs about responsibility, beliefs about the allocation of responsibility, and beliefs about how others allocate responsibility were used to evaluate interpersonal influences on responsibility. Whereas personal beliefs about responsibility were related to all OCD symptom types, it appears that beliefs about other people's responsibility were related to only a subgroup of symptom types. Furthermore, the belief that one is more responsible than others predicts OC symptoms beyond commonly assessed personal beliefs about responsibility. Finally, individuals with OC symptoms tend to allocate more responsibility to themselves than others, compared to individuals without OC symptoms, despite the fact that individuals with OC symptoms believe that others tend to allocate responsibility equitably. Results are discussed in terms of cognitive models of OCD.
\end{abstract}

Keywords: Obsessive-compulsive disorder, responsibility, interpersonal beliefs, symptoms, beliefs.

\section{Introduction}

Obsessive Compulsive Disorder (OCD) is characterized by intrusive thoughts, images, or impulses that are distressing and unacceptable, and/or repetitive behaviour that is performed to reduce anxiety and/or prevent some dreaded event from occurring (American Psychiatric Association, 2000). Cognitive-behavioural models of OCD posit that it is not the content of an intrusive thought per se that motivates an individual to neutralize the thought, but rather the significance attached to that thought that motivates efforts to neutralize (Salkovskis, 1985). Six belief domains that are believed to play a significant role in the development and maintenance of OC symptoms have been identified (Obsessive Compulsive Cognitions Working Group (OCCWG), 1997, 2003, 2005), one of which is an inflated sense of responsibility.

Salkovskis (1985) proposed that intrusive thoughts are interpreted by an individual with OCD as indicating that the individual may be responsible to prevent harm from occurring. As a result of this interpretation, the individual experiences a negative mood and is motivated to

Reprint requests to Adam S. Radomsky, Concordia University, 7141 Sherbrooke Street West, Montreal, Quebec, H4B 1R6, Canada. E-mail: adam.radomsky@concordia.ca 
take action to prevent harm from occurring, thus driven to perform some neutralizing action or compulsion. This in turn reduces anxiety, and temporarily eliminates the intrusion, reinforcing the neutralizing action. Inflated responsibility was later operationally defined as "the belief that one possesses pivotal power to provoke or prevent subjectively crucial negative outcomes. The outcomes may be actual, that is having consequences in the real world and/or at a moral level" (Rhéaume, Ladouceur, Freeston and LeTarte, 1995). According to this definition, if someone anticipates the outcome of an event to have negative consequences and believes s/he has the power to prevent the occurrence of this outcome, s/he will feel responsible to take action to prevent that negative outcome from occurring.

Numerous studies support the idea that an inflated sense of responsibility for negative events is related to OCD. Several researchers have developed questionnaires designed to measure responsibility (e.g. Salkovskis et al., 2000; OCCWG, 1997, 2001, 2003, 2005; Wilson and Chambless, 1999). Based on these questionnaires, individuals meeting DSM-IV criteria for OCD score higher on inflated responsibility than individuals with other anxiety disorders and normal controls (Salkovskis et al., 2000; Steketee, Frost and Cohen, 1998). Additionally, Steketee and colleagues (1998) have demonstrated that individuals with other anxiety disorders are not significantly different from control participants on measures of inflated responsibility, suggesting that inflated responsibility is likely a unique feature of OCD.

Other researchers have attempted to manipulate responsibility in an effort to demonstrate that inflated responsibility can either induce or exacerbate symptoms of OCD. Lopatka and Rachman (1995) had participants who met the diagnostic criteria for OCD sign a contract giving full responsibility to participants (i.e., Increased Responsibility) or giving full responsibility to the experimenter (i.e., Decreased Responsibility) for the outcome of a check. Participants were asked to check something that would normally induce compulsive checking. Compared to a control condition where there was no responsibility manipulation, Lopatka and Rachman (1995) found that low responsibility resulted in significant decreases in perceived discomfort, the urge to check, the estimated probability and seriousness of anticipated harm, and the estimated probability and seriousness of anticipated criticism. In contrast, increased responsibility resulted in a significant increase in the likelihood of anticipated criticism, but there were no significant differences in perceived discomfort, urge to check, or the estimated probability and seriousness of anticipated harm compared to a control condition.

Shafran (1997) replicated these findings, demonstrating that the urge to neutralize, perceived discomfort and the probability of threat were greater under conditions of high responsibility (i.e., when the experimenter was absent from the room), compared to conditions of low responsibility (i.e., when the experimenter was present in the room). Furthermore, using a similar manipulation to Lopatka and Rachman (1995), Radomsky, Rachman and Hammond (2001) demonstrated that under conditions of high responsibility individuals with OCD exhibit a larger memory bias for threat-relevant information, and show less memory confidence than under conditions of low responsibility or no responsibility. Furthermore, Bouchard, Rhéaume, and Ladouceur (1999) observed that increasing responsibility resulted in increased hesitations and checking during a pill sorting task in a non-clinical sample of individuals. This suggests that inflated responsibility can lead to OC symptoms and can exacerbate OCD in diverse ways, ranging from symptom severity (e.g., urges to check) to information processing (e.g., memory and metamemory).

Although an inflated sense of responsibility indeed appears to be an important component of OCD, research is increasingly demonstrating that inflated responsibility is comprised of 
many components. For example, Salkovskis and colleagues (2000) developed two separate responsibility questionnaires to measure different aspects of responsibility. The Responsibility Attitude Scale (RAS) was designed to assess general beliefs about responsibility. The Responsibility Interpretations Questionnaire (RIQ) was designed to assess beliefs about responsibility related to specific intrusive thoughts and the frequency with which these beliefs occur. Salkovskis and colleagues (2000) found that although the two questionnaires were significantly correlated with one another, regression analyses demonstrated that each questionnaire contributed uniquely to the prediction of OCD symptomatology. Furthermore, a third questionnaire, the Responsibility Appraisals Questionnaire (RAQ), developed by Rachman, Thordarson, Shafran and Woody (1995), assesses beliefs about responsibility related to responsibility for harm, responsibility about social contexts, a positive outlook on responsibility, and thought-action fusion. However, it was found that perhaps only thoughtaction fusion appears to be related to OCD symptoms.

Menzies, Harris, Cumming and Einstein (2000) recently examined responsibility for negative outcomes for the self versus for others. They asked a group of undergraduate students to rate the perceived severity and probability of 10 negative outcomes. Half of the participants completed a version of the questionnaire in which the participant was performing the actions and was therefore personally responsible for the outcome, and the remaining participants completed a version of the questionnaire in which someone else was performing the actions and therefore the participant was not personally responsible for the outcome. Observing a nonclinical sample, these researchers found that if one is personally responsible for preventing a negative outcome, the perceived severity of the outcome is significantly greater than if someone else is responsible for preventing the outcome. These results suggest that the perception of personal responsibility may be different than the perception of responsibility in others.

It may be that beliefs about how responsible other people are may influence one's personal responsibility for an event. That is, if one believes that other people are not responsible, one may feel it is necessary to increase personal responsibility and to thus engage in neutralizing behaviour to prevent harm. Beliefs about how other people think about responsibility may also influence one's personal sense of responsibility. If a person believes that other people expect him/her to be very responsible, that person may feel very responsible. Furthermore, personal responsibility for the outcome of an event may also be influenced by beliefs about how others distribute responsibility. That is, if one believes that other people expect them to be responsible, they may actually feel more responsible to prevent harm. The purpose of this study is to examine in greater detail how these interpersonal aspects of responsibility are related to symptoms of OCD.

Three different questionnaires were developed to assess three aspects of responsibility related to the perceptions of other people. The first questionnaire assessed thoughts about other people's beliefs about responsibility. The second questionnaire assessed comparative beliefs about responsibility. That is, whether an individual feels more or less responsible compared to other people. The final questionnaire assessed beliefs about how responsibility is distributed between the self and another person for positive, negative, and neutral events. In addition to personal beliefs about the distribution of responsibility, this questionnaire also assessed beliefs about how others distribute responsibility. It was hypothesized that OC symptoms would be associated with the belief that one is more responsible than other people. It was further predicted that individuals scoring high on a measure of OC symptoms, compared to those scoring low on a measure of OC symptoms, would allocate more responsibility to themselves than to others, 
and that they would expect others to also allocate a significant proportion of responsibility to the individual with OC symptoms. In general, we were interested in determining how these various interpersonal beliefs about responsibility are related to symptoms of OCD and how they relate to one's personal sense of responsibility.

\section{Method}

\section{Participants}

Two hundred and one undergraduate students participated in the study. One participant was eliminated from analysis due to missing data. The mean age of participants was 23.28 ( $S D=$ 5.02) years, with a range from 17 to 54 years. There were 164 female participants and 36 male participants.

\section{Measures}

Vancouver Obsessive Compulsive Inventory (VOCI). The VOCI (Thordarson et al., 2004) is a 55-item self-report questionnaire that assesses a variety of symptoms associated with OCD. The VOCI was used to assess OCD symptomatology in the participants, and to separate participants into high and low checking groups. Along with a total score, the VOCI also contains six subscales, each assessing a specific symptom area of OCD: contamination, checking, obsessions, hoarding, "just rightness", and indecisiveness.

The VOCI exhibits good internal consistency and good test-retest reliability within a clinical population of people with OCD. Good internal consistency has been established in a student population; however, while test-retest reliability within a student population was initially shown to be poor, it has recently been shown to be excellent (Radomsky et al., 2005). Furthermore, the VOCI exhibits good convergent and divergent validity in both a clinical and student population.

Beck Depression Inventory - II (BDI-II). The BDI-II (Beck, Steer and Brown, 1996) is a 21-item self-report questionnaire measuring depressive symptomatology and was used as a measure of depression for this study. The BDI-II exhibits high internal consistency and excellent test-retest reliability. Furthermore, this measure demonstrates good convergent and divergent validity.

Responsibility Attitude Scale (RAS). The RAS (Salkovskis et al., 2000) is a 26-item selfreport questionnaire designed to assess general attitudes and beliefs about responsibility. The RAS has good internal consistency, with an alpha of .92 reported in the original validation study. Furthermore, it demonstrates good test-retest reliability. The RAS demonstrates acceptable predictive validity, with individuals with OCD scoring higher on the RAS than individuals without OCD. Lower scores on the RAS indicate more responsibility.

\section{New measures}

Responsibility Attitude Scale-Others. ${ }^{1}$ The RAS-Others is a questionnaire that is modelled after the RAS. Each item of the RAS was reworded so that the questions asked about others beliefs about responsibility rather than personal believes about responsibility. For example, item 1 on the RAS, "I feel responsible for things that go wrong", was reworded for the RAS-Others 
to say "Other people feel responsible for things that go wrong". Each item is rated on a scale from $1=$ Totally agree to $7=$ Totally disagree, with lower scores indicating greater responsibility. As with the RAS, lower scores on the RAS-Others indicate the belief that others are more responsible.

Comparative Beliefs About Responsibility Scale. ${ }^{1}$ The CBARS is a 24-item questionnaire designed for this study to examine if OCD symptomatology is related to beliefs about how responsible one is relative to other people. Items were generated by reviewing and rewording items from other responsibility questionnaires such as the RAS. For example, Item 5 on the CBARS is "Other people are not as responsible as I am". Each item is rated on a scale from $0=$ Disagree strongly to $4=$ Agree strongly.

Distribution of Responsibility Questionnaire. ${ }^{1}$ The DORQ is a 6-item scale designed to examine how people think responsibility is allocated for a variety of events of different valences. For each question, the respondent answers using a Visual Analog Scale (VAS) with lower scores indicating more allocation of responsibility to the respondent, higher scores indicating more allocation of responsibility to others, and intermediate scores indicating equal allocation of responsibility between the respondent and other people. For negative, neutral, and positive events, the respondent is asked to rate how s/he would allocate responsibility, as well as to rate how s/he thinks other people would allocate responsibility.

\section{Procedure}

Participants completed a questionnaire package that included the questionnaires described above. They were requested to complete the questionnaires in one sitting and had their name entered in a draw for one of five cash prizes as compensation for their participation.

\section{Results}

\section{People's beliefs about responsibility}

Reliability of the RAS-Others. The RAS was used to assess personal beliefs about responsibility, and the RAS-Others was used to assess the perception of other people's beliefs about responsibility. Because the RAS-Others was a modified version of the RAS, Cronbach's alpha was calculated to ensure that the scale was reliable. Cronbach's alpha for RAS-Others was .93. The correlation of the RAS and RAS-Others was $r=.45, p<.0001$, suggesting that although these scales are related, they likely measure different constructs.

Relationship of RAS-Others to OC symptoms. To examine how beliefs about personal responsibility and the perception of other people's beliefs about responsibility are related to symptoms of OCD, correlations between the RAS and RAS-Others with the BDI-II, VOCI total and subscale scores were calculated. Table 1 shows the means of the RAS and RAS-Others as well as the Pearson correlations with the BDI-II, VOCI total and subscales.

As expected, the RAS was significantly negatively correlated with the total VOCI score and all VOCI subscales, as well as with the BDI-II (i.e., lower scores, indicating more responsibility, are associated with OC symptoms and depression). The RAS-Others was also

\footnotetext{
${ }^{1}$ The questionnaires are available by request from the 3rd author, ASR.
} 
Table 1 Means and standard deviation of the RAS, and RAS-Others, and the correlation of the RAS and RAS-Others with symptoms of OCD

\begin{tabular}{lccc}
\hline & & & $\begin{array}{c}\text { RAS-Others - } \\
\text { Partial correlation } \\
\text { controlling for RAS }\end{array}$ \\
\hline Mean & RAS & RAS-Others & 119.98 \\
Standard Deviation & 113.76 & $(21.63)$ & \\
\hline Correlations & $(22.87)$ & & \\
BDI-II & $-.42^{* *}$ & -.07 & $.16^{*}$ \\
VOCI-total & $-.60^{* *}$ & $-.16^{*}$ & -.06 \\
Checking & $-.36^{* *}$ & $-.22^{*}$ & .07 \\
Contamination & $-.38^{* *}$ & $-.22^{*}$ & .04 \\
Obsessions & $-.52^{* *}$ & -.01 & $.18^{* *}$ \\
Hoarding & $-.49^{* *}$ & $-.19^{*}$ & $.28^{* *}$ \\
Just Right & $-.48^{* *}$ & -.08 & \\
Indecisiveness & $-.56^{* *}$ & -.05 & \\
\hline${ }^{* *} p<.001 ;{ }^{*} p<.05$. & & &
\end{tabular}

significantly negatively correlated with the total VOCI score and the Contamination, Checking, and Hoarding subscales of the VOCI, but not the "Just right", Indecisiveness, or Obsessions subscales. The RAS-Others was also unrelated to the BDI-II.

Interestingly, after controlling for RAS, the pattern of relationships between RAS-Others and OC symptoms changed. Partial correlations between the RAS-Others and the VOCI, controlling for the RAS, were calculated. When the RAS was controlled for, the RAS-Others was significantly positively correlated with the VOCI total score, as well as the "Just right", Indecisiveness, and Obsessions subscales of the VOCI (i.e., these OC symptoms were related to beliefs that other people are not responsible, since higher scores on the RAS-Others indicate the belief that others are less responsible). After controlling for RAS, the RAS-Others was no longer significantly related to the contamination, checking or hoarding subscales of the VOCI.

\section{Comparative Beliefs about Responsibility}

Reliability of the CBARS. The Cronbach's alpha for the CBARS was .89, and the item-total correlations for the 24 items ranged between -.08 and .65. The following items had low or negative item-total correlations: "Other people are more likely than I am to check that the lights are turned off at the end of the day", "I trust other people to make sure that the faucet in the kitchen is not leaking", and "Other people are more likely than I am to make sure that the window is locked". These items were therefore eliminated from the scale. After these items were eliminated, Cronbach's alpha for the CBARS rose to .91, with item-total correlations ranging between .37 and .65. Thus the 21-item CBARS has acceptable reliability. The mean CBARS score was 25.39, with a standard deviation of 13.46 .

The relationship of comparative beliefs to symptoms of OCD. To examine how the CBARS is related to symptoms of OCD, correlations between the CBARS and VOCI total and subscale 
Table 2 The relationship between the CBARS and the RAS, BDI-II, and VOCI

\begin{tabular}{lcc}
\hline & \multicolumn{2}{c}{ CBARS } \\
\cline { 2 - 3 } Variable & Correlations & $\begin{array}{c}\text { Partial correlations } \\
\text { controlling for RAS and BDI-II }\end{array}$ \\
\hline RAS & $-.59^{* *}$ & \\
BDI-II & $.35^{* *}$ & $.17^{*}$ \\
VOCI-total & $.49^{* *}$ & $.12^{\dagger}$ \\
Checking & $.31^{* *}$ & $.17^{*}$ \\
Contamination & $.36^{* *}$ & .04 \\
Obsessions & $.38^{* *}$ & -.10 \\
Hoarding & $.26^{* *}$ & $.23^{* *}$ \\
Just Right & $.50^{* *}$ & .08 \\
Indecisiveness & $.42^{* *}$ & \\
\hline${ }^{*} p<.05 ;{ }^{* *} p<.01 ;{ }^{\dagger} p<.10$. &
\end{tabular}

scores were calculated. As Table 2 demonstrates, the CBARS was significantly correlated with the VOCI total score as well as all subscale scores and the RAS. As is also shown in Table 2, the CBARS was significantly correlated with the BDI-II, indicating that comparative beliefs about responsibility are also related to symptoms of depression.

We were concerned that the CBARS may be related to OC symptoms only through its relationship with the RAS and the BDI-II. To control for beliefs about responsibility and depression, we computed partial correlations between the VOCI and its subscales and the CBARS, controlling for the RAS and the BDI-II. As Table 2 demonstrates, CBARS was significantly correlated with the VOCI total score, Contamination subscale, and the "Just right" subscale even after controlling for the RAS and BDI-II. The relationship between the VOCI checking subscale and the CBARS approached significance. The correlations between the VOCI Obsessions, Hoarding, and Indecisiveness subscales and the CBARS were no longer significant.

\section{Distribution of responsibility}

To examine how the distribution of responsibility is related to OC symptoms, participants were divided into high and low OC symptom groups based upon VOCI total scores. Participants with VOCI total scores in the top quintile were classified as High OC symptoms $(n=76)$, and the remaining participants were classified as Low OC symptoms $(n=123)$. Table 3 demonstrates that there were no differences between High and Low OC symptom groups in relation to gender, $\chi^{2}(d f=1)=.41, p=.52$; however, there were significant differences between the groups in age as well as on scores on the BDI-II, and VOCI total and subscale scores, $t \mathrm{~s}(197)>3.03, p \mathrm{~s}<.003$.

Each item on the DORQ was considered separately. A repeated measures ANOVA with valence (positive vs. negative vs. neutral), and allocator (self vs. other) as within participant factors, and OC symptom group (high vs. low) as the between participant factor was calculated. There was a significant main effect for allocator, $F(1,194)=17.59, p<.001$, 
Table 3 Differences between High and Low OC symptom groups

\begin{tabular}{lcc}
\hline Measures & High OC symptom & Low OC symptom \\
\hline$N$ & 76 & 123 \\
Age & $21.93^{* *}$ & 24.11 \\
& $(2.82)$ & $(5.85)$ \\
$\%$ Female & $84 \%$ & $81 \%$ \\
VOCI total & $50.25^{* *}$ & 10.88 \\
& $(23.82)$ & $(7.00)$ \\
Checking & $4.69^{* *}$ & 0.56 \\
& $(5.60)$ & $(1.35)$ \\
Contamination & $9.18^{* *}$ & 2.00 \\
& $(7.20)$ & $(2.89)$ \\
Obsessions & $8.60^{* *}$ & 1.40 \\
& $(7.75)$ & $(2.16)$ \\
Hoarding & $7.38^{* *}$ & 2.12 \\
& $(4.26)$ & $(2.75)$ \\
Just Right & $12.23^{* *}$ & 2.60 \\
& $(6.82)$ & $(2.25)$ \\
Indecisiveness & 8.18 & 2.21 \\
& $(4.74)$ & $(2.10)$ \\
BDI-II & $(16.70$ & 6.25 \\
& $(11.67)$ & $(5.30)$ \\
\hline$* * *$
\end{tabular}

indicating that, in general, participants allocated more responsibility to themselves, whereas they believe others tend to allocate responsibility more equitably regardless of the valence of the event. The main effect of valence was also significant, $F(2,388)=13.74, p<.0001$. Follow-up paired comparisons revealed that that individuals tend to allocate the most responsibility to themselves for neutral events, followed by bad events, and tend to allocate responsibility for good events equitably. The main effect of OC group was not significant, $F(1,194)=2.54, p=.11$; however, the valence $\times$ OC group interaction was significant, $F(2,388)=4.89, p<.01$. High OCs allocated significantly more responsibility to themselves for neutral events compared to low OCs, $p<.01$. There was a non-significant trend for High OCs, compared to Low OCs, to allocate more responsibility to themselves for negative events, $p<.10$, and good events, $p<.10$, as well.

Since the allocator $\times$ OC group interaction approached significance, $F(1,194)=2.90$, $p=.09$, it was decided to conduct follow-up pairwise comparisons. There were no differences between the groups in terms of how they thought other people allocate responsibility, $p=.77$; however, for personal allocation of responsibility, the high OCs tended to allocate more responsibility to themselves compared to low OCs, $p=.02$. Neither the valence $\times$ allocator interaction, $F(2,388)=.50, p=.61$, nor the three-way allocator $\times$ valence $\times$ OC group interaction, $F(2,388)=1.47, p=.23$, were significant. In summary, as demonstrated in Figure 1, people tended to allocate the most responsibility to themselves for neutral events, and the most responsibility to others for positive events. However, in relation to neutral events, high OCs allocated more responsibility to themselves as compared to low OCs. Overall, people 


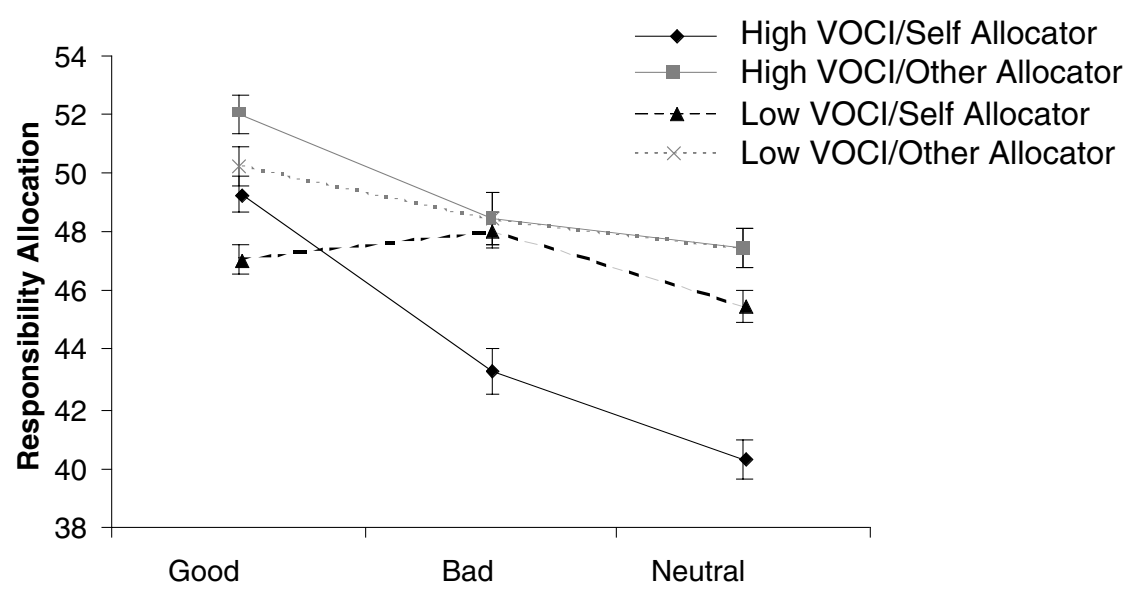

Figure 1 The allocation of responsibility for good, bad, and neutral events in groups reporting high and low OC symptoms. Lower scores indicate more allocation of responsibility to the respondent

allocated more responsibility to themselves, whereas they expected that other people would allocate responsibility more equitably.

\section{Discussion}

The results of this study suggest that the relationships between inflated responsibility and OC symptomatology would benefit from consideration in greater detail. In general, the results of this study suggest that: a) beliefs about personal responsibility and beliefs about others' responsibility are related to OC symptoms in different ways, as assessed by the RAS and RASOthers; b) the belief that one is more responsible than other people predicts OC symptoms beyond just personal beliefs about responsibility, as assessed by the CBARS; c) how one allocates responsibility for an event between oneself and others predicts OC symptoms, as assessed by the DORQ; and that d) this effect appears to be influenced by whether the event is positive, negative, or neutral, as assessed by the DORQ.

\section{Personal responsibility versus the responsibility of others}

We found that both personal beliefs about responsibility and beliefs about the responsibility of others were significantly related to symptoms of OCD. Interestingly, these relationships appeared to be different.

Consistent with numerous other studies (e.g., Salkovskis et al., 2000), an inflated sense of personal responsibility was strongly related to all measured symptoms of OCD. In contrast, initially, the belief that other people are also very responsible was correlated with symptoms of OCD that are externally manifested, such as checking, contamination, and hoarding. However, the relationship between these OC symptoms and the belief that others are responsible disappeared after personal beliefs about responsibility were accounted for. That is, for symptoms of checking, contamination and hoarding, where other people may have an 
impact, the perception of how responsible other people are appears to be intertwined with personal feelings of responsibility.

An entirely different pattern emerged when we examined internally manifested OC symptoms, such as obsessions, an inability to make decisions, and the need to attain an internal sense of "just rightness". We found that these symptoms were unrelated to the beliefs about how responsible other people are when personal beliefs about responsibility were not accounted for. However, after controlling for personal beliefs about responsibility, the belief that other people are not responsible was significantly related to internal symptoms of OCD, as well as overall symptoms of OCD. This suggests that individuals with obsessions and compulsions that are less influenced by the actions of others clearly separate their beliefs about their own responsibility and their beliefs about other people's responsibility. Individuals with externally related obsessions may take on more responsibility because they believe that others are not as responsible. However, for individuals with internally related obsessions, it may be that the responsibility of other people is not as relevant since other people cannot influence the individual's thoughts.

\section{Comparative beliefs}

The examination of comparative beliefs about responsibility suggests that inflated responsibility may be structured differently in association with different OC symptoms. The sense that one is more responsible compared to other people was significantly related to all symptoms of OCD. However, after controlling for depression and inflated personal responsibility, only OC symptoms related to contamination, checking, and "just rightness" were related to the feeling that one is more responsible compared to others.

The different pattern of partial correlations found for comparative beliefs and found for beliefs about how responsible other people are suggests that the structure of inflated responsibility is also complex. The differentiation between internal symptoms and external symptoms of OCD found when we examined beliefs about how responsible other people are was not evident when we examined comparative beliefs about responsibility. Rather, the belief that one is more responsible compared to other people appears to be most related to symptoms where one is attempting to ensure that harm is prevented or that an action is completed to satisfaction. In contrast, OC symptoms such as obsessions and difficulty making decisions are least related to beliefs that one is more responsible than other people. If one believes that $\mathrm{s} / \mathrm{he}$ can prevent harm or ensure an action has been completed properly better than other people, one may feel more responsible to make sure that harm is prevented or the action is completed. On the other hand, if one is continually questioning one's actions one may feel responsible for the outcome of an event, but may not necessarily think that they are responsible. Therefore, comparative beliefs about responsibility may be less relevant for these types of OC symptoms.

\section{Distribution of responsibility}

We found distinct differences in the way individuals distribute responsibility for different events and the way in which they believe other people distribute responsibility. In general, people tend to allocate more responsibility to themselves than they do to other people. In contrast, people believe that others allocate responsibility equitably. Furthermore, the valence of the 
event appeared to influence the way that responsibility is distributed, regardless of whether the distribution is made by oneself or others. Responsibility is most equitably distributed for good events, whereas most of the responsibility for the outcome of an event when it is neutral is given to the individual rather than to other people. The distribution of responsibility for negative events appears to be somewhere between the distribution of responsibility for good events and neutral events.

The way in which valence influenced the allocation of responsibility was different for individuals with OC symptoms. Specifically, compared to individuals with fewer OC symptoms, individuals with high OC symptoms appear to allocate more responsibility to themselves than others for both negative and neutral events. However, individuals with high OC symptoms allocate responsibility similarly to those with fewer OC symptoms when the outcome of the event is good. The fact that individuals with OC symptoms take more responsibility for negative events is consistent with the current definition of inflated responsibility, "the belief that one possesses pivotal power to provoke or prevent subjectively crucial negative outcomes" (Rhéaume et al., 1995). These results suggest that not only do individuals feel more responsible to prevent a negative outcome, but that they also believe that others are less responsible for that negative outcome.

The fact that individuals with OC symptoms also allocate more responsibility to themselves than others for neutral events suggests that they may define what constitutes a negative event differently than individuals without OC symptoms. Specifically, individuals with OC symptoms may have a more rigid definition for the valence of an outcome such that events may be only negative or positive. Furthermore, their threshold for defining what constitutes a negative event may be lower, such that even neutral events become negative. Consistent with this explanation, other studies have demonstrated that checkers tend to feel more responsible for moderate risk and low risk situations compared to control participants (Foa, Sacks, Tolin, Przeworski and Amir, 2002), suggesting that they have a lower threshold for what constitutes a risky event. Our results suggest that it may not only be the degree of risk that influences responsibility but also the perceived outcome of the situation; such that individuals with OC symptoms feel the least responsible when the outcome of an event is good, but take more responsibility when the outcome of an event is bad or neutral.

\section{Concluding remarks}

Understanding the various components of inflated responsibility in greater detail may help improve cognitive-behavioural treatments of OCD. This study suggests that inflated responsibility may be targeted by reframing how responsibility is allocated. In the same way that one can demonstrate the bias towards thought-action fusion for negative thoughts and the absence of thought-action fusion for positive events, one could examine whether it makes sense for an individual to allocate more responsibility to him/herself for bad events, but not for good events. Furthermore, one could also point out the discrepancy between personal distribution of responsibility and the belief about how others distribute responsibility. Individuals who believe that others allocate responsibility to them would likely benefit from different interventions specifically tailored to these beliefs.

This study also suggests that a fine-grained analysis of how responsibility beliefs differ according to OC symptoms may also help improve treatment strategies. For example, separating personal feelings of responsibility from beliefs about how responsible others are 
may help decrease inflated responsibility in checkers, washers and hoarders, but may be less relevant to inflated responsibility for individuals with obsessions. While discussions about symptom subtype development are being pursued, caution is recommended when interpreting findings based solely upon symptoms (McKay et al., 2004).

This study used a non-clinical sample, and therefore the clinical implications of these findings should be interpreted with caution. However, a review examining non-clinical samples in OCD research did conclude that clinical and non-clinical OC samples are similar across many different dimensions (Gibbs, 1996). Future research should examine interpersonal beliefs in individuals with diagnosed OCD in order to establish that biases demonstrated in this study do indeed exist in clinical populations. Furthermore, it should also be noted that this study is based on questionnaires, and thus subject to participant bias. Experimental research manipulating interpersonal beliefs about responsibility would be useful to determine how such beliefs influence personal responsibility and OC symptoms, such as the urge to check.

This study, along with others (e.g. Foa et al., 2002; Menzies et al., 2000) points to the need to examine inflated responsibility in greater detail. The purpose of this study was to investigate an aspect of inflated responsibility that has not previously been examined. Results of this study suggest that interpersonal aspects of responsibility, including beliefs about how responsible others are, comparative beliefs about responsibility, and the distribution of responsibility between oneself and others, are related to symptoms of OCD in complex ways that would benefit from additional investigation.

\section{Acknowledgements}

Portions of these data were presented at the 2003 Association for the Advancement of Behaviour Therapy convention in Boston, MA, as well as at the 2004 conference of the European Association for Behavioural and Cognitive Therapies in Manchester, England. This study was funded in part by a Fonds québécois de la recherche sur la société et la culture Nouveau Chercheur award and by a Canadian Institutes of Health Research New Investigator award held by the third author.

\section{References}

American Psychiatric Association (2000). Diagnostic and Statistical Manual of Mental Disorders (4th Ed-Test Revision). Washington DC: Author.

Beck, A. T., Steer, R. A. and Brown, G. K. (1996). Beck Depression Inventory Manual (2nd ed.). San Antonio TX: Psychological Corporation.

Bouchard, C., Rhéaume, J. and Ladouceur, R. (1999). Responsibility and perfectionism in OCD: an experimental study. Behaviour Research and Therapy, 37, 239-248.

Foa, E. B., Sacks, M. B., Tolin, D. F., Przeworski, A. and Amir, N. (2002). Inflated perception of responsibility for harm in OCD patients with and without checking compulsions: a replication and extension. Journal of Anxiety Disorders, 16, 443-453.

Gibbs, N. A. (1996). Nonclinical populations in research on obsessive-compulsive disorder: a critical review. Clinical Psychology Review, 16, 729-773.

Lopatka, C. and Rachman, S. (1995). Perceived responsibility and compulsive checking: an experimental analysis. Behaviour Research and Therapy, 33, 673-684. 
Menzies, R. G., Harris, L. M., Cumming, S. R. and Einstein, D. A. (2000). The relationship between inflated personal responsibility and exaggerated danger expectancies in obsessive-compulsive concerns. Behaviour Research and Therapy, 38, 1029-1037.

McKay, D., Abramowitz, J. S., Calamari, J. E., Kyrios, M., Radomsky, A., Sookman, D., Taylor, S. and Wilhelm, S. (2004). A critical evaluation of obsessive-compulsive disorder subtypes: symptoms versus mechanisms. Clinical Psychology Review, 24, 283-313.

Obsessive Compulsive Cognitions Working Group (1997). Cognitive assessment of obsessivecompulsive disorder. Behaviour Research and Therapy, 35, 667-681.

Obsessive Compulsive Cognitions Working Group (2001). Development and initial validation of the obsessive beliefs questionnaire and the interpretation of intrusions inventory. Behaviour Research and Therapy, 39, 987-1006.

Obsessive Compulsive Cognitions Working Group (2003). Psychometric validation of the Obsessive Beliefs Questionnaire and the Interpretation of Intrusions Inventory: Part 1. Behaviour Research and Therapy, 41, 863-878.

Obsessive Compulsive Cognitions Working Group (2005). Psychometric validation of the Obsessive Beliefs Questionnaire and the Interpretation of Intrusions Inventory: Part 2, Factor analyses and testing of a brief version. Behaviour Research and Therapy, 43, 1527-1542.

Rachman, S., Thordarson, D. S., Shafran, R. and Woody, S. R. (1995). Perceived responsibility: structure and significance. Behaviour Research and Therapy, 33, 779-784.

Radomsky, A. S., Rachman, S. and Hammond, D. (2001). Memory bias, confidence and responsibility in compulsive checking. Behaviour Research and Therapy, 39, 813-822.

Radomsky, A. S., Ouimet, A. J., Ashbaugh, A. R., Lavoie, S. L., Parrish, C. L. and O'Connor, K. P. (2005, under review). Psychometric properties of the French and English versions of the Vancouver Obsessional Compulsive Inventory and the Symmetry, Ordering and Arranging Questionnaire.

Rhéaume, J., Ladouceur, R., Freeston, M. H. and Letarte, H. (1995). Inflated responsibility in Obsessive compulsive disorder: validation of an operational definition. Behaviour Research and Therapy, 33, 159-169.

Salkovskis, P. M. (1985). Obsessional-compulsive problems: a cognitive-behavioural analysis. Behaviour Research and Therapy, 23, 571-583.

Salkovskis, P. M., Wroe, A. L., Gledhill, A., Morrison, N., Forrester, E., Richards, C., Reynolds, M. and Thorpe, S. (2000). Responsibility attitudes and interpretations are characteristic of obsessive compulsive disorder. Behaviour Research and Therapy, 38, 347-372.

Shafran, R. (1997). The manipulation of responsibility in obsessive-compulsive disorder. British Journal of Clinical Psychology, 36, 397-407.

Steketee, G., Frost, R. O. and Cohen, I. (1998). Beliefs in obsessive-compulsive disorder. Journal of Anxiety Disorders, 12, 525-537.

Thordarson, D. S., Radomsky, A. S., Rachman, S., Shafran, R., Sawchuk, C. N. and Hakstian, A. R. (2004). The Vancouver Obsessional Compulsive Inventory (VOCI). Behaviour Research and Therapy, 42, 1289-1314.

Wilson, K. A. and Chambless, D. L. (1999). Inflated perceptions of responsibility and obsessivecompulsive symptoms. Behaviour Research and Therapy, 37, 325-335. 